\title{
Chen-Mobius voice communication system
}

\author{
Sa Yuchao ${ }^{\mathrm{a}}$, He Wei ${ }^{\mathrm{b}^{*}}$ \\ Yunnan Normal University physics and electronic information college Yunnan, Radio Physics \\ *Corresponding author: he99wei@aliyun.com
}

Keywords: Chen-Mobius communication system, Voice signal, Cycle quadratic function waveform

\begin{abstract}
The result of cycle quadratic function waveform Chen-Mobius transformation is applied to voice communication system. We compute the two classes of mutually orthogonal sets of functions, then we took them as a new type of modulation and demodulation function, and we found itself these two functions are orthogonal, but the function groups to which they belong are not orthogonal. A new voice communication system is constituted by them. In this paper, we use cycle quadratic function waveform to modulate and demodulate the voice communication systems, and the system was simulated by use matlab platform. We made the corresponding tables, in which there are effects of different periods demodulation signal for the voice communication system. And the calculated results were compared. Chen-Mobius voice signal to the system is a useful complement, and it complements modulation and demodulation function group too.

A famous scholar, now Chinese Academy of Sciences, Tsinghua University, professor Chen Nanxian uses infinite series Mobius inversion formula to solve a number of important applications of inverse problems in physics. This kind of work in 1990 got the whole version in famous "NATURE" magazine, it also got special assessment and highly valued. Su Wuxun, Zhang Weibin of Huaqiao University Professor put the method of Mobius transformation into several common waveform(periodic rectangular pulse, parity symmetry square wave and triangular wave) inverse Fourier transform operation Series, got expansions of Sine function, cosine function and various common signal waveforms, they obtained function family that is orthogonal to function family of various common waveform signal, last used them to calculate the various coefficient of expansion and demodulate the information. Then they applied them to the communication system, proposed a new communication system. That is the new Chen-Mobius communication systems. This paper is an extension of signal modulation and demodulation, we added quadratic function and makes Chen-Mobius system more perfect, and you can use more modem signal.
\end{abstract}

\section{Mobius transformation theory}

Mobius Function. In number theory, Mobius function is defined as:

$$
\mu(\mathrm{n})=\left\{\begin{array}{cc}
1 & \mathrm{n}=1 \\
(-1)^{\mathrm{r}} & \mathrm{n} \text { is the product of different } \mathrm{r} \text { prime Numbers } \\
0 & \text { Could be divided by the square of a prime number }
\end{array}\right.
$$

Mobius function has an important feature is

$$
\sum_{\text {din }} \mu(d)=\left\{\begin{array}{cc}
1 & n=1 \\
0 & n=2,3,4 \ldots
\end{array}\right.
$$

It's integral symbol for each $n$ that means the sum of positive integer factor $d$ (including 1 and $n$ ).

$$
\begin{aligned}
& \text { For example, } \\
& \mathrm{n}=1, \quad \sum_{\mathrm{d} / 1} \mu(\mathrm{d})=\mu(1)=1 \\
& \mathrm{n}=2, \sum_{\mathrm{d} / 2} \mu(\mathrm{d})=\mu(1)+\mu(2)=0 \\
& \mathrm{n}=3, \sum_{\mathrm{d} / 2} \mu(\mathrm{d})=\mu(1)+\mu(3)=0 \\
& \mathrm{n}=4, \quad \sum_{\mathrm{d} / 2} \mu(\mathrm{d})=\mu(1)+\mu(2)+\mu(3)=0
\end{aligned}
$$

For the convenience of description, we introduced a concept of complete product: for any two positive integers, if the arithmetic function $f$ is not zero at the same time and $f(m n)=f(m) f(n)$, it 
means $\mathrm{f}$ is complete the product.

Mobius Inversion Formula. In classical number theory, the famous Mobius inversion formula is: for any positive integer $n, F(x)$ is defined on the set of natural numbers arithmetic function, and it satisfies:

$$
\mathrm{G}(\mathrm{n})=\sum_{\mathrm{dn}} \mathrm{F}(\mathrm{n})
$$

$$
\text { then } \quad F(n)=\sum_{d i n} \mu(d) G\left(\frac{n}{d}\right) \text {. }
$$

Wherein the integral sign expressed the summation of all positive whole numbers of $n, \mu(n)$ is the Mobius function.

Professor Chen Nanxian promotes $F(n)=\sum_{d n} \mu(d) G\left(\frac{n}{d}\right)$ as a new function F (x). If F (x) is a continuous function of the variable $\mathrm{x}$, and satisfies:

$$
\mathrm{G}(\mathrm{x})=\sum_{\mathrm{n}=1}^{\infty} \mathrm{F}\left(\frac{\mathrm{x}}{\mathrm{n}}\right)
$$

then $\mathrm{F}(\mathrm{x})=\sum_{\mathrm{n}=1}^{\infty} \mu(\mathrm{n}) \mathrm{G}\left(\frac{\mathrm{x}}{\mathrm{n}}\right)$.

The promotion provides a tool to solve physics’ and other disciplines’ inverse problem.

\section{Voice communication's simulation}

Matlab Simulation Of Analog Voice Communications. Matlab voice communication simulation is shown below:

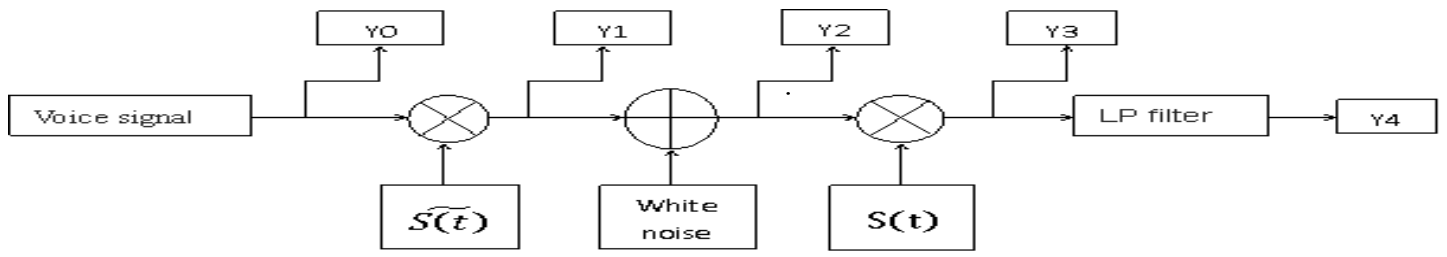

Fig1 Matlab voice communication simulation

Figure's Signal is inputs voice signal. $\widetilde{S(t)}$ is a quadratic function of the inverse transform wave and trapezoidal wave, $S(t)$ is a quadratic function wave. Analog voice transmission simulation process is described as follows:

(1)Voice signal inputs: $\mathrm{Y} \times \widetilde{\mathrm{S}(\mathrm{t})}=\mathrm{Y} 1(\mathrm{t})$;

(2) $\mathrm{Y} 1(\mathrm{t})$ and the noise signal are added: $\mathrm{Y} 1(\mathrm{t})+\mathrm{n}(\mathrm{t})=\mathrm{Y} 2(\mathrm{t})$;

(3) $\mathrm{Y} 2(\mathrm{t}) \times \mathrm{S}(\mathrm{t})=\mathrm{Y}(3)$; (4) $\mathrm{Y} 3(\mathrm{t})$ by the final filter output $\mathrm{Y} 4(\mathrm{t})$;

(5)Y1, Y2, Y3, Y4 were observed by using the oscilloscope waveforms.

This system uses a random noise $n(t)$, the voice signal power Ps $=917.9807$.

Calculation Of Digital Signal Inverse Transform . For even symmetric functionF(t), it can be expressed as a Fourier series: $\mathrm{F}(\mathrm{t})=\mathrm{a} 0+\sum_{\mathrm{n}=\mathrm{i}}^{\infty} \mathrm{r}(\mathrm{n}) \cos (\mathrm{n} \omega \mathrm{t})$. We know $\mathrm{S}(\mathrm{T})=\sum_{\mathrm{n}=\mathrm{i}}^{\infty} \mathrm{r}(\mathrm{n}) \cos (\mathrm{n} \omega \mathrm{t})$. For odd symmetric functions, $\mathrm{S}(\mathrm{T})=\sum_{\mathrm{n}=\mathrm{i}}^{\infty} \mathrm{r}(\mathrm{n}) \sin (\mathrm{n} \omega \mathrm{t}), \mathrm{S}(\mathrm{T})$ is nonpositive cosine terms. We converted $\mathrm{S}(\mathrm{t})$ to $\widetilde{S(t)}$ by using Mobius inverse transformation. $\mathrm{S}(\mathrm{T})$ is a single periodic function of $\mathrm{S}(\mathrm{t}) \cdot \widetilde{\mathrm{S}(\mathrm{t})}=\sum_{\mathrm{m}: \mathrm{k}} \mathrm{I}\left(\frac{\mathrm{k}}{\mathrm{m}}\right) \cos (\mathrm{m} \omega \mathrm{t})$ or $\widetilde{\mathrm{S}(\mathrm{t})}=\sum_{\mathrm{m}: \mathrm{k}} \mathrm{I}\left(\frac{\mathrm{k}}{\mathrm{m}}\right) \sin (\mathrm{m} \omega \mathrm{t})$, Each summation symbol represents a summation of the $\mathrm{m}$ of the $\mathrm{k}$. Wherein the value of $\mathrm{I}(\mathrm{k})$ calculated by Fourier series, the formula is $\sum_{n: k} I(k) r\left(\frac{k}{n}\right)$ can be shown that $\mathrm{S}(\mathrm{t})$ and $\widetilde{\mathrm{S}(\mathrm{t})}$ are orthogonal. Is the so-called orthogonal 
$\frac{1}{\pi} \int_{0}^{2 \pi} \mathrm{S}(\mathrm{t}) \widetilde{\mathrm{S}(\mathrm{t})} \mathrm{d}(\mathrm{t})=\delta_{\mathrm{l}}$.

Calculation and simulation of cycle quadratic function's inverse transformation Periodic Quadratic Function

$$
\mathrm{S}(\mathrm{T})=\mathrm{t}^{2}, \quad(-0.0001 \pi \leq \mathrm{t} \leq 0.0001 \pi) .
$$

Function image are as follows:

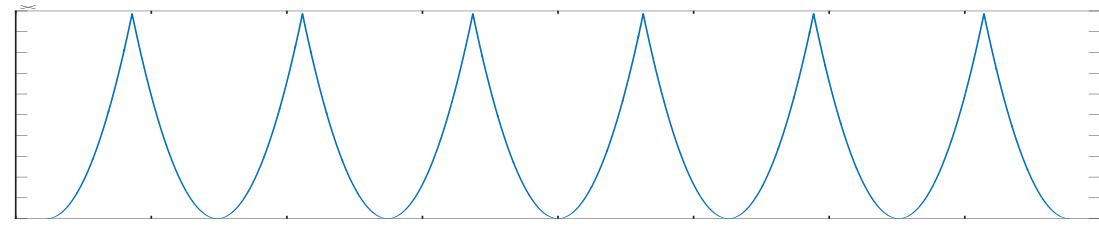

Fig1. Cycle Periodic Quadratic Function

Cycle quadratic function wave Fourier series, Chen - Mobius inverse transformation conversion

$$
S(T)=\sum_{n=1}^{\infty}\left[\frac{\frac{n^{2} \pi^{2} \sin (n \pi)}{2}-\sin (n \pi)+n \pi \cos (n \pi)}{250000 n^{3} \pi}\right] \cos (n \omega t) \text {. }
$$

From the above equation:

$$
r(n)=\frac{\frac{n^{2} \pi^{2} \sin (n \pi)}{2}-\sin (n \pi)+n \pi \cos (n \pi)}{250000 n^{3} \pi} \text {. }
$$

Where $\sum_{\mathrm{n} / \mathrm{k}}^{\infty} \mathrm{I}(\mathrm{n}) \mathrm{r}\left(\frac{\mathrm{k}}{\mathrm{n}}\right)=\delta_{\mathrm{k} \mathrm{l}}$. It can be calculated I (n) value. By the inverse transformation formula $\widetilde{\mathrm{S}(\mathrm{t})}=\Sigma_{\mathrm{m}: \mathrm{k}} \mathrm{I}\left(\frac{\mathrm{k}}{\mathrm{m}}\right) \cos (\mathrm{m} \omega \mathrm{t})$, determined $\widetilde{\mathrm{S}(\mathrm{t})}$.

You can see that the inverse transformation function only has a finite number of cosine combinations. In the traditional model, the two orthogonal modulation and demodulation functions are the same family of function, this function is orthogonal to the family itself. Chen - Mobius system of modulation and demodulation functions are different, even though they are mutually orthogonal function, but each function group itself are orthogonal. For such applications, they have many advantages, they calculate the amount of relatively traditional analog systems to reduce the number, and have a better confidentiality.

\section{Comparison Of The Original Signal And Modulation Signals After Demodulation}

(1) contrast of 3 order after the inverse transformation demodulation signal

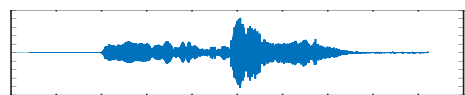

Fig. 2 The original Voice signal

(2) contrast of 5 order after the inverse transformation demodulation signal

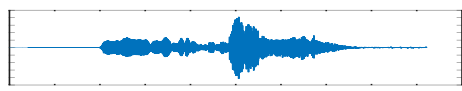

Fig. 4 The original Voice signal

(3) contrast of 10 order after the inverse transformation demodulation signal

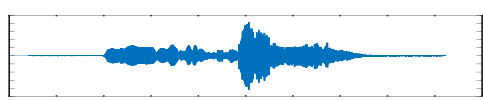

Fig. 6 The original Voice signal

Fig. 7 Modulation signals after demodulation

(4) contrast of 15 order after the inverse transformation demodulation signal 


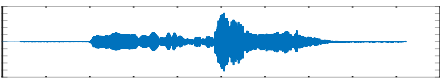

Fig. 8 The original Voice signal

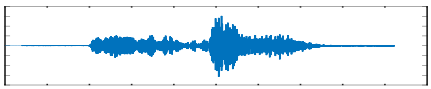

Fig. 9 Modulation signals after demodulation

Table1. The corresponding signal to noise ratio of each order

\begin{tabular}{llll}
\hline System order $[\mathrm{Hz}]$ & Signal power $[\mathrm{Hz}]$ & Noise power $[\mathrm{Hz}]$ & SNR $[\mathrm{Hz}]$ \\
\hline 3order & 917.9807 & 9.2145 & 19.9836 \\
5 order & 917.9807 & 9.2140 & 19.9838 \\
10 order & 917.9807 & 9.2479 & 19.9679 \\
15 order & 917.9807 & 9.1723 & 20.0036 \\
\hline
\end{tabular}

Through the above graphical analysis of results: quadratic function Chen-Mobius system is feasible. We Compared system of 3, 10 and 15 rank-order Mobius inverse transform, found when you select 15, the system works best.
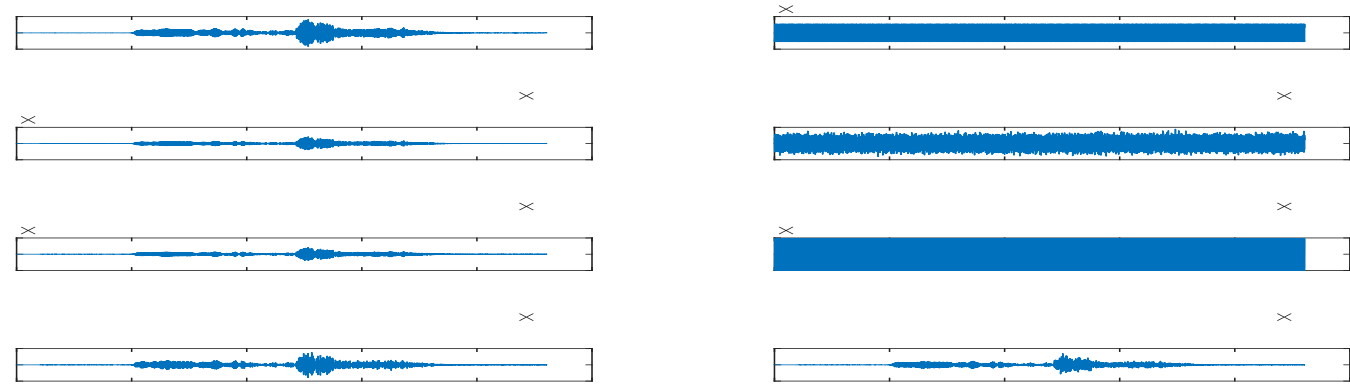

Fig. 10 The system diagram of the cycle of $0.0001 \pi$
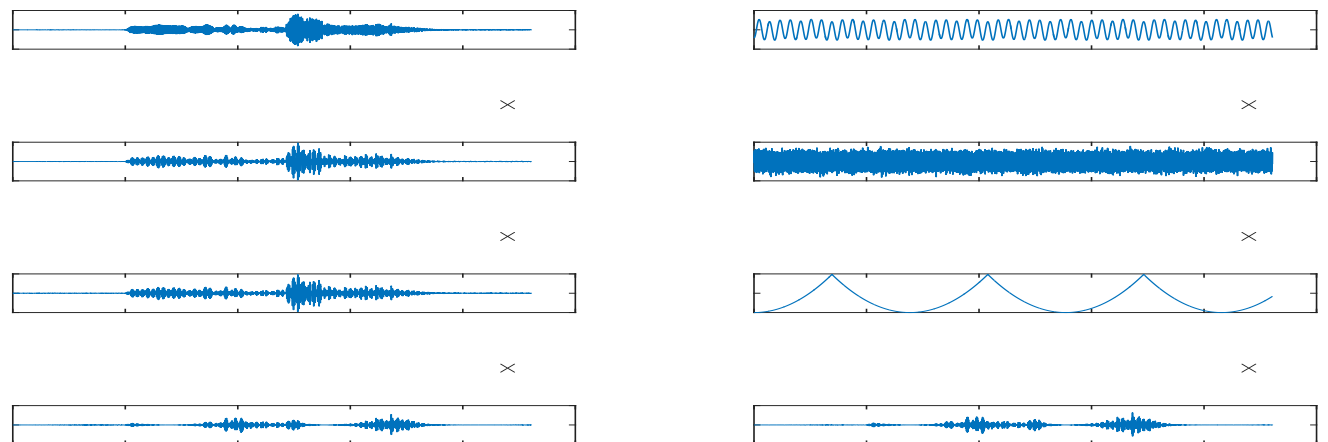

$\times$

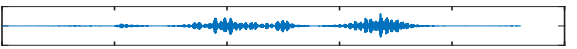

Fig.11 The system diagram of the cycle of $0.1 \pi$

Compare the above picture, we find that a period of a quadratic function and its inverse transform $0.1 \pi$ wave, which corresponds to the input waveform and the final system vary widely, analyze the causes can know the length of the sound time of 3.1 seconds, and the use of sampling frequency $\mathrm{f}=44100 \mathrm{~Hz}$ we demodulation function of frequency closer to the audio sampling frequency, the better, the more the demodulated audio, because of the time matlab calculations, we just take a $10000 \mathrm{~Hz}$.

\section{Conclusion}

We may safely draw the conclusion from the above simulation: Cycle quadratic function and it's Chen - Mobius inverse transformation can be used for voice signal system. Had been studied the rectangle, triangle, sawtooth, this paper is supplement of Chen - Mobius system, increased the quadratic function. We can see that the period of modulation and demodulation system is influential, 
the smaller the period, we can see that the closer the output and input. In summary Chen-Mobius communication system for voice transmission is significant.

\section{Acknowledgements}

This project was supported by National Natural Science programs (51267021) and College students' innovative entrepreneurial training programs (2015).

\section{References}

[1] Chen Nanxian, Modified Mobius inverse formula and Its applications in physics [J].Phys Rev Lett, 1990, 64 (11) 1193-1195

[2] Chen Nanxian, Li Gangying, Theoretical investigation on the inverse black-body Radiation problem [J].IEEE T Antenn Propag, 1990, 38(8): 1287-1290

[3] Rosu H, Mobius inverse problem for distorted black holes ( II ) [J]. Nuovo Cimento, 1993, 108B(12): 1333-1339

[4] Xie Tading, Goldsmi THP F, A new method for analyzing IRAS data to determine the dust temperature distribution [J]. Astrophys J, 1991, 371: 31-34

[5] Wang Jieming, Temperature distribution of accretion disks in active galacticNuclei[J].Astrophys J, 1996, 469: 564-571

[6]Su wuxun et al, "The evaluations of the inverse transform of eight often-used waveforms by Mobius transform---the inverse transform of their Fourier series” [J].CHINESE JOURNAL OF ELECTRONICS, 2005, 14(3): 513-518 